These cases usually expose carotid artery, jugular vein dura and have a high rate of recurrence.

Videos will be provided for each pathology showing the technique of removal and hearing reconstruction.

doi:10.1017/S0022215116002814

\section{Cholesteatoma in Children (N715)}

\section{ID: 715.2}

Long term results of total ossiculoplasty in pediatric cholesteatoma surgery

Presenting Author: Francoise Denoyelle

Francoise Denoyelle ${ }^{1}$, Jerome Nevoux ${ }^{2}$, Pierre Chauvin ${ }^{3}$, Noël Garabédian ${ }^{1}$

${ }^{1}$ Necker Children's Hospital, APHP and Paris

Descartes University, Paris France, ${ }^{2}$ Hopitel de

Bicêtre et Université Paris XI, ${ }^{3}$ Department of

Public Health, Saint Antoine Hospital ans Paris VI University

Learning Objectives: To evaluate the long-term results and predictive factors of a good outcome with the use of a total ossicular replacement prosthesis in pediatric cholesteatoma surgery.

Objective: To evaluate the long-term results and predictive factors of a good outcome with the use of a total ossicular replacement prosthesis in pediatric cholesteatoma surgery.

Design and setting: Retrospective case review in a tertiary referral center.

Patients: The study included 114 children (116 ears).

Interventions: A total of 116 ears underwent total ossicular chain reconstruction with a titanium prosthesis. Cartilage was always used for tympanic membrane reconstruction.

Main Outcome Measures: Audiological results were evaluated according to the guidelines of the American Academy of Otolaryngology-Head and Neck Surgery. Predictive factors of audiological results were determined. Logistic regression and $\mathrm{X} 2$ tests were used for statistical analysis.

Results: The mean age at surgery was 9.8 years. Ossiculoplasty was performed during second-look surgery in 91 ears $(78.4 \%)$ and during another stage in 25 ears $(21.6 \%)$. The first-stage procedure was always performed for cholesteatoma. Audiometric results were available for 116 ears at 1 year, for 89 ears $(76.7 \%)$ at 2 years, and for 42 ears $(36.2 \%)$ at 5 years. Closure of the average air-bone gap (ABG) to within $20 \mathrm{~dB}$ was achieved in 65 ears $(56 \%)$ at 1 year. The mean (SD) preoperative and postoperative (at 1 year) ABGs were $41.0(9.5) \mathrm{dB}$ and 22.4 (12.6) dB, respectively. There were no cases of extrusion, but 17 luxations of the prosthesis were confirmed by computed tomography. Luxation occurred on average at 31.4 months. Three $4000-\mathrm{Hz}$ degradations of bone conduction were reported, with no dead ears. We examined 3 predictive factors of auditory results: preoperative $\mathrm{ABG}$, footplate status, and postoperative otoscopic findings.

Conclusions: Total ossiculoplasty is a reliable technique in children. Long-term hearing outcomes are stable and satisfactory, but luxation can occur at any time. Preoperative $\mathrm{ABG}$ and footplate status are negative predictive factors of auditory results.

doi:10.1017/S0022215116002826

\section{Ventilation and Gas exchange in middle ear (R716)}

\section{ID: 716.1}

Middle ear pressure maintenance:

1) a concert played by many instruments.

2) Pathology as compensation.

Presenting Author: Udi Cinamon

Udi Cinamon

Wolfson Medical Center

Learning Objectives: Middle ear pressure maintenance: 1) a concert played by many instruments. 2) Pathology as compensation.

It is crucial that the pressure in the middle ear (ME) will be kept circa to ambient pressure. As a physiological system that needs to confront constant intrinsic and extrinsic changes (e.g., cardiovascular system, respiratory system, etc.) the $\mathrm{ME}$ requires to possess special capabilities to maintain a physiological steady state. This ME pressure homeostasis is a concert played by several mechanisms, i.e., pressure regulators meant to neutralize or minimize pressure changes. Adjusting the amount of gas, its flow and diffusion, as well as the volume of the middle ear cleft, temperature all mechanism that follow the law of gases $[\mathrm{PV}=\mathrm{nRT}]$ :

Volume: Size matters, the mastoid and the tympanic membrane (TM) being a "pressure buffers".

Surface matters: The "radiator" effect of the ME cleft regarding gas exchange, temperature, moisture (number of molecules, Temperature). The Eustachian tube (ET) being a conduit possessing a pumping effect. Nerves and pressure receptors may control ventilation by opening the ET. When one or more of these mechanisms fails a "disorder" may kick-in. Therefore, developing a chronic ME insufficiency is accepted as the patho-physiological setting for developing chronic ME disease, clinically presented as otitis media with effusion, atelectasis of the TM or associated with developing cholesteatoma.

These chronic changes can be addressed as compensatory mechanisms (e.g., heart hypertrophy to keep-up with perfusion having a failing heart). Edema of the mucosa, engorgement of vessels and transudate will diminish the volume and influence gaseous content in the ME elevating pressure. TM atelectasis changes the ME volume being a pressure buffer.

Failure to confront a prompt and significant pressure change, a situation in which the ME pressure needs to be elevated instantly, will be presented as barotrauma. The consequence would be transudate, hemorrhage, and TM perforation all means "trying" to compensate. 